\title{
Periodic and Aperiodic Bunching in the Addition Spectra of Quantum Dots
}

\author{
N.B. Zhitenev and R.C. Ashoori \\ Department of Physics, Massachusetts Institute of Technology, Cambridge, Massachusetts, 02139 \\ L.N. Pfeiffer and K.W. West \\ Bell Labs., Lucent Technologies, Murray Hill, NJ 07974
}

\begin{abstract}
We study electron addition spectra of quantum dots in a broad range of electron occupancies starting from the first electron. Spectra for dots containing $<200$ electrons reveal a surprising feature. Electron additions are not evenly spaced in gate voltage. Rather, they group into bunches. With increasing electron number the bunching evolves from occurring randomly to periodically at about every fifth electron. The periodicity of the bunching and features in electron tunneling rates suggest that the bunching is associated with electron additions into spatially distinct regions within the dots.
\end{abstract}

PACS 71.30.+h, 73.20.Jc, 73.20Dx, 73.40.Gk

Coulomb blockade $(\mathrm{CB})$ is one of the most fundamental and robust concepts in mesoscopic physics. Lambe and Jaklevic first made this point clear in a seminal experiment nearly 30 years ago [1]. For a metallic island poorly coupled to its surroundings, the number of electrons is quantized at low temperatures. Due to the repulsive Coulomb potential created by electrons already on the island, the energy required to add an electron to the island increases by a fixed amount $\Delta$ with each electron added. An external gate electrode capacitively coupled to the island through a capacitance $C_{g}$ can be used to cause electrons to transfer on and off the island. Additions of single electrons occur periodically in gate voltage with a period $e / C_{g}$. Physical phenomena in the system with characteristic energy scales on order $\Delta$ can disrupt the periodicity. For instance superconductivity in the system, can cause electrons to be added as periodically occurring pairs [2]. One does not expect such results in a semiconductor or a normal metal sample.

In a semiconductor system containing enough electrons to be considered metallic, the deviations from exact periodicity in gate voltage are expected to be rather weak. The corresponding small parameter is $r_{s} / R$, where $r_{s}$ is the screening length (about $100 \AA$ ), and $R$ is a characteristic size of the system $(0.2-1 \mu \mathrm{m})[3]$. In the opposite limit of a disordered mesoscopic system containing a few electrons localized at spatially distinct sites, significant fluctuations in the addition spectrum are predicted [6].

Several years ago, one experiment on a semiconductor system displayed results which appeared to violate CB [7]. Electrons were seen to enter a quantum dot in pairs rather than individually. The system was a twodimensional dot with a $1 \mu \mathrm{m}$ diameter. It was somewhat atypical of semiconductor quantum dot experiments: the first electrons to enter this system occupy random potential minima created by disorder, and the different minima were screened from each other by a metallic electrode which was only a few hundred Angstroms away. Nonetheless, the exact coincidence of electron additions into the system is quite a surprise. The experiment used a method called single electron capacitance spectroscopy (SECS) and was unique in allowing study of electron additions into separate localized sites.

This letter describes results from a systematic SECS study of different sized two-dimensional dots to help determine the origin of the strange correlation in electron additions. We found that in dots with lithographic diameters larger than $0.4 \mu \mathrm{m}$ containing small numbers of electrons, electron additions are sometimes grouped in bunches comprising from 2 to 6 electrons. The exact pairing seen previously [7] is apparently a limiting case of this more general tendency.

As the electron occupancy $(\mathrm{N})$ is increased in dots of all sizes, the bunching eventually ceases, and a periodic Coulomb blockade spectrum develops. However, application of magnetic field causes the bunching effect to reappear. Surprisingly, in dots with diameters of 0.4-0.5 $\mu \mathrm{m}$, the bunching occurs periodically with electron number. Approximately every fifth electron addition peak pairs with a neighboring peak. The details of the addition spectra yield critical clues about the nature of the bunching.

A schematic of our samples is shown on inset to Fig.1b. They are similar to the ones described in Refs. [7,8]. The $\mathrm{AlGaAs} / \mathrm{GaAs}$ wafer contains the following layers (from the bottom to the top): $3000 \AA n^{+}$GaAs, $400 \AA$ GaAs spacer layer, $136 \AA$ AlGaAs/GaAs superlattice tunnel barrier, $175 \AA$ GaAs quantum well, $500 \AA$ AlGaAs blocking barrier, $300 \AA$ GaAs cap layer. A mesa with deep ohmic contacts down to $n^{+}$GaAs is initially defined. Then a circular $\mathrm{Cr} / \mathrm{Au}$ gate electrode is fabricated on the top of the mesa. 8 dots were studied with gate diameters ranging from $1.6 \mu \mathrm{m}$ to $0.2 \mu \mathrm{m}$. Plasma etching produces a short pillar (300 $\AA$ tall) using the gate electrode as a mask. Electrons remain in the quantum well only in the region below the pillar [7]. A larger overlapping metal electrode then provides electrical connection to the gate. The measurements are carried out using an on-chip bridge circuit described in [7].

Fig.1a displays the electron addition spectrum at zero 
magnetic field for a dot of $500 \mathrm{~nm}$ lithographic diameter. For gate biases below $-500 \mathrm{mV}$, the quantum dot is empty. Peaks occur in the capacitance at gate voltages for single electron additions to the quantum dot [7]. Fig. 1b displays the Fourier transform (FT) of the measured capacitance for successive gate voltage intervals of equal length. For large N (bottom traces), only a single dominant frequency component is present in the spectrum. The position of the FT peak counts the number of electron additions per gate voltage interval. As the gate bias is made more negative, fewer electrons are added in a gate voltage interval, reflecting a decrease in the $C_{g}$ due to lateral contraction of the electron droplet. At voltages more negative than $-400 \mathrm{mV}$, the single peak evolves into a broad low frequency spectrum. The broadening indicates that the gate voltage spacings between electron additions become uneven.

The peak positions in Fig.1b can be recalculated to determine the area of the dot using a simple parallel plate capacitor model. This scale is shown on the top axis. Altogether, we can distinctly resolve about 600 electron additions in this dot. The gate voltage scale can be directly converted to an energy scale $\Delta E=\alpha \Delta V_{g}$ with the lever-arm $\alpha \sim 0.5$ for these structures determined from the geometry of the dot as described in [7]. The gate voltage position of the Nth capacitance peak, when multiplied by the lever arm, directly measures the chemical potential $\mu_{N}$ of the dot containing $\mathrm{N}$ electrons [3].

The magnetic field evolution of a portion of the electron addition spectrum is shown in Fig.2a. The grayscale map displays the first 150 additions, with capacitance peaks visible as black traces. Examination of the bottom of Fig.2a shows that the first 7 electrons enter the dot at widely spaced voltages. They may enter into a single potential minimum or minima spaced closely enough that the Coulomb repulsion between the sites is sufficient to keep the peaks widely spaced. Beyond the 7 th electron trace, something extraordinary occurs. Three electrons enter the dot in very rapid succession in gate voltage over the full range of magnetic fields. The next two electrons also join in a bunch (pair). For higher $\mathrm{N}$, other bunches can be seen. We note that the experiment shows no hysteretic effects. The bunching is a phenomenon which occurs with the dot in equilibrium with its surroundings.

After about 40 electrons are added to the dot, the bunching develops into a periodic pattern, with one bunch appearing for each 4-6 electrons added to the dot. As $\mathrm{N}$ is increased beyond about 80 , the bunching ceases for zero magnetic field. Instead, the electron additions occur with nearly perfect periodicity, as is typical of $\mathrm{CB}$. However, for nonzero magnetic field strengths, the bunching phenomenon returns. Bunches again occur periodically in gate voltage, and the period is about the same as that for the zero field bunches. A zoom-in of this behavior is shown in Fig. 2b. The onset of bunching shifts to larger magnetic fields with increasing concentration, and the bunches are no longer observable at fields up to 13 Tesla for more than about 200 electrons in the dot.

The behavior of each electron trace can be described roughly as follows. The magnetic field at which all electrons fall into the lowest Landau level, $\nu=2$, can be readily identified as a maximum in the traces at around $\mathrm{B}=2 \mathrm{~T}[10]$. As in two-dimensional systems the chemical potential peaks just as higher Landau levels depopulate completely. Jumps in the traces at higher magnetic fields, where both spin levels of the lowest Landau level are filled, are usually interpreted as single electron spinflips 9, 10]. The flatness of the traces around $\mathrm{B}=6 \mathrm{~T}$ demarcates total spin polarization of the dot. We refer to this range as the vicinity of $\nu=1$. For higher fields, the traces rise nearly linearly with magnetic field.

A bunched pair of traces in Fig.2b is marked with $\mathrm{a} *$. These traces are fairly representative of all of the other traces which appear as electron pairs. Starting at some nonzero magnetic field the two traces are seen to stick together but then they split as the field approaches that which yields $\nu=1$. Passing through $\nu=1$, the lower trace of the bunched pair splits from the trace above it, only to join with the trace below it.

There is a region devoid of electron additions in the spectrum on both sides of any bunch. The mean interval between electron additions hence remains the same on a larger scale, even though individual traces have bunched. This is clearly noticeable for small N. At large N, Fig. 2b reveals that the spacing between the non-bunched additions at nonzero magnetic field is larger than the spacing at zero field where the bunching phenomenon is not present.

The bunching appears to be a universal behavior. We have seen the bunches in all investigated quantum dots with lithographic diameters greater than $0.4 \mu \mathrm{m}$. At low $\mathrm{N}$, the bunching emerges without any apparent pattern. With increasing $\mathrm{N}$, the spacing between peaks becomes regular at zero magnetic field, but application of sufficiently strong field revives the bunching. The boundary for the onset of the bunching is remarkably similar for all dots in which bunches are observed, regardless of their size. This boundary moves to higher magnetic fields as the average electron density (note, not $\mathrm{N}$ ) in the dot is increased roughly according to the linear relation

$$
n_{\text {onset }}=(1.1+0.08 \times B[\text { Tesla }]) \times 10^{11} \mathrm{~cm}^{-2}
$$

The nearly periodic bunching (pairing) pattern is observed for dots created with lithographic diameters of about $0.5 \mu \mathrm{m}$. For larger dots the bunching still occurs as the magnetic field surpasses the threshold (1), but the bunches appear to occur randomly with gate voltage rather than periodically.

The bunching phenomenon is reflected in the rate at which the electrons tunnel into the dot. At zero magnetic field, the rate of electron tunneling between the $n^{+}$ 
substrate and the quantum well is about $5 \mathrm{MHz}$. Measurements at a much lower frequency of $\mathrm{f}=200 \mathrm{KHz}$ are only sensitive to the tunneling resistance if the tunneling is strongly suppressed by electrons correlations within the dot [8,10]. At very low temperatures $(\mathrm{T}<0.1 \mathrm{~K})$ the tunneling rate drops substantially in particular regions of magnetic field and electron occupancy.

Figure 2c shows a measurement of the addition spectrum of the same dot at base temperature $\mathrm{T}=30 \mathrm{mK}$ after thermal cycling up to room temperature. The details of the addition spectrum of the dot are modified but the overall bunching behavior remains qualitatively unchanged. For low N, shown on bottom part of Fig. $2 c$, contrast in all electron traces is the same over the entire range of magnetic field, indicating that the electron tunneling rate is much larger than the measurement frequency. The middle segment of Fig.2c displays the capacitance spectrum in a range of larger $\mathrm{N}$ (75-95 electrons in the dot). Notice here that some of the traces extinguish as magnetic field increases. As the peaks diminish in strength, the phase of the electron tunneling signal lags relative to the ac excitation [8]. This detectable decay of the tunneling rates begins in the vicinity of $\nu=1$, for sufficiently large number of electrons in the dot.

The only traces observable at the highest magnetic field of $\mathrm{B}=13 \mathrm{~T}$ in Fig.2c extend from paired traces. Examination of the intensity and phase of these unextinguished traces shows that they typically result from only a single electron rather than two electrons tunneling. We note that the dc bias in the experiment is adjusted very slowly so that the electron occupancy in the dot changes even though peaks are not seen in the capacitance experiment. Finally, at higher N (Fig. 2c, upper part), the bunching disappears, and all traces extinguish equally.

We believe that pairs of electrons in the quantum dot observed previously by Ashoori et al. [7] are a special case of the bunches in the regime of electrons strongly localized within a large $(1 \mu \mathrm{m}$ lithographic diameter $)$ dot. In dots of similar size, we have seen more examples of bunches with the traces of two and sometimes three electrons that exactly overlap over a range of magnetic fields. In general, paired traces from dots with smaller lithographic diameters do not coincide exactly. Two theoretical models have been suggested to explain the origin of the pairs. In one model [1], lattice deformation mediates the effective attraction in the pairs. In another model [12], redistribution of electrons in the dot makes the energy for adding two electrons less than twice the energy for adding a single electron. Both of these models predict a dramatic suppression of the tunneling rate as soon as two electrons are joined into a pair, since both electrons must be added into the dot in a coherent fashion. Having studied a large number of exact pairs in the frequency range $50 \mathrm{KHz}-1 \mathrm{MHz}$ we have never observed a significant drop of the tunneling rate when the traces merge. This suggests that the paired electrons tunnel into the dot independently, though they are added to the system at precisely the same gate voltage. Remarkably, the data indicate that filling one state of a pair has no effect on the energy of the other state in the pair.

A coarse comparison of addition spectra at small and large $\mathrm{N}$ leads us to conclude that the bunches are intrinsically associated with electron localization within the quantum dot. The strong fluctuation of the spacings between electron additions seen at low $\mathrm{N}$ (though not the pairing) is expected in the strongly localized regime [6]. The development of a periodic CB spectrum visible at higher $\mathrm{N}$ at zero magnetic field indicates the metallic character of the dot in this regime. The periodic bunching occurs in the transition range between these regimes.

The reproducibility of the periodic bunching pattern in several dots cannot be ascribed to a peculiarity of the disorder potential. As we observe the same 4-5 electron periodicity of the pairing over the entire magnetic field range, we start our analysis from a consideration of electrons in a dot in infinite magnetic field. Electrons can then be considered as classical point charges. In a twodimensional system of uniform potential, classical point charges rest on a triangular lattice. When the lattice is restricted within a quantum dot, mostly the outer row of electrons is deformed to conform to the cylindrical symmetry of the confining potential [13, 14]. Electrons in the center of the dot maintain the triangular lattice arrangement. The sequence of the electron entrances into the classical dot can be calculated. Levitov [14] demonstrated recently that for 50-150 electrons in the dot, one electron of each 4-5 electrons added enters the outer row (circumference). This coincides with the frequency of the periodic bunches in our data. Intriguingly, though the classical point charges cannot describe the electron density in zero magnetic field, the same periodicity of electron bunching is experimentally observed down to zero magnetic field.

The idea that one of the bunched electrons appears at the edge of the electron droplet is consistent with the contrast observed in the tunneling rate. The drop of the tunneling rate for $\nu<1$ illustrated on Fig.2c indicates poor overlap of the $\mathrm{N}$ - electron ground state with the $(N+1)$ - electron ground state in the dot. Such a decay of the tunneling rate can be considered as a special case of the Coulomb gap observed for larger systems 15

- The origin of the tunneling suppression can be understood semi-classically. The tunneling process suddenly adds one more electron into a dot. Until the system relaxes to its new ground state, the tunneling process is not finished. Hence, the effective tunnel barrier depends on the disturbance of the density distribution and its relaxation rate. The higher tunneling rate observed for one trace in each bunch can be explained if that electron is introduced into the edge of the dot. That electron then has fewer neighbors (and they are farther away) in comparison with an electron introduced into the bulk of the 
dot

The mechanism creating the bunching phenomenon remains highly speculative. What can compete with the usually dominant Coulomb addition energy to disturb the addition spectrum so profoundly? Hartree-Fock calculations demonstrate 16 that exchange can mediate a local attraction between electrons, tending to keep the system compact. The $\nu=1$ state is believed to be fully spin-polarized, and exchange maintains $\nu=1$ as the lowest energy state of the system over a range of magnetic fields [16]. The switching of the bunches at $\nu=1$ (Fig.2b) appears to effectively broaden this range for some of the traces suggesting the involvement of the exchange interaction in bunch formation.

We gratefully acknowledge numerous useful discussions with Leonid Levitov. Expert etching of samples was performed by S.J. Pearton and J.W. Lee. This work is supported by the ONR, the Packard Foundation, JSEP-DAAH04-95-1-0038, NSF DMR-9357226 and DMR-9311825, and DMR-9421109.

[1] J. Lambe, and R.C. Jaklevic, Phys. Rev. Lett., 22, 1371 (1969).

[2] M.T. Tuominen, et al., Phys. Rev. Lett., 69, 1997 (1992). P. Lafarge, et al., Phys. Rev. Lett., 70, 994 (1993).

[3] H.van Houten, C.W.J. Beenakker, and A.A.M. Staring, in Single Charge Tunneling, H. Grabert and M.H. Devoret, Editors. 1992, Plenum Press: New York. p. 167216.

[4] U. Sivan, et al., Phys. Rev. Lett., 77, 1123 (1996).

[5] R. Berkovits, and B.L. Altshuler, Phys. Rev. B, 55, 5297 (1997).

[6] A. A. Koulakov, F.G. Pikus, and B.I. Shklovskii, preprint cond- mat/9612236, 1997.

[7] R. C. Ashoori, et al., Phys. Rev. Lett., 68, 3088 (1992).

[8] R. C. Ashoori, et al., Phys. Rev. Lett., 71, 613 (1993).

[9] P. L. McEuen, et al., Phys. Rev. B, 45, 11419 (1992); O. Klein, et al., Phys. Rev. Lett., 74, 785 (1995).

[10] R. C. Ashoori, Nature, 379, 413 (1996).

[11] Y. Wan, G. Ortiz, and P. Phillips, Phys. Rev. Lett., 75, 2879 (1995).

[12] M. E. Raikh, L.I.Glazman, and L.E. Zhukov, Phys. Rev. Lett., 77, 1354 (1996).

[13] V. M. Bedanov, and F.M. Peeters, Phys. Rev. B, 49, 2667 (1994). F. M. Peeters, V.A. Schweigert, and V.M. Bedanov, Physica B, 212, 237 (1995).

[14] L.S. Levitov, private communication.

[15] R. C. Ashoori, et al., Phys. Rev. B, 48, 4616 (1993). R. C. Ashoori, et al., Phys. Rev. Lett., 64, 681 (1990). J. P. Eisenstein, L.N. Pfeiffer, and K.W. West, Phys. Rev. Lett., 69, 3804 (1992).

[16] A H. MacDonald, S.R.E. Yang, and M.D. Johnson, Aust. J.Phys, 46, 345 (1993). C. de Chamon, and X.G. Wen, Phys. Rev. B, 49, 8227 (1994).
FIG. 1. a) Quantum dot capacitance as a function of gate voltage. The peak at $-500 \mathrm{mV}$ denotes the appearance of the first electron in the dot. $\mathrm{T}=0.3 \mathrm{~K}$. b) Fourier transform spectra of measured capacitance for succesive gate voltage intervals of $28 \mathrm{mV}$ starting from $-500 \mathrm{mV}$ at the top to $-80 \mathrm{mV}$ at the bottom. Peaks in spectra correspond to the number of electrons added into the dot per given voltage interval. Inset: the scheme of the dot.

FIG. 2. Grayscale image of the measured capacitance. Black denotes capacitance peaks. Electron occupancies are indicated as numbers. a) Vertical axis - gate voltage ranging from $-511 \mathrm{mV}$ (bottom) to $-328 \mathrm{mV}$ (top). $\mathrm{T}=0.3 \mathrm{~K} . \mathrm{b}$ ) Zoom - in of spectrum surrounded by box in a). c) Segments of the addition spectrum measured after thermally cycling the dot to room temperature at $\mathrm{T}=50 \mathrm{mK}$. Vertical bar corresponds to energy change of $5 \mathrm{meV}$ calculated from the gate voltage using constant geometrical factor (common for all images in c) ). 


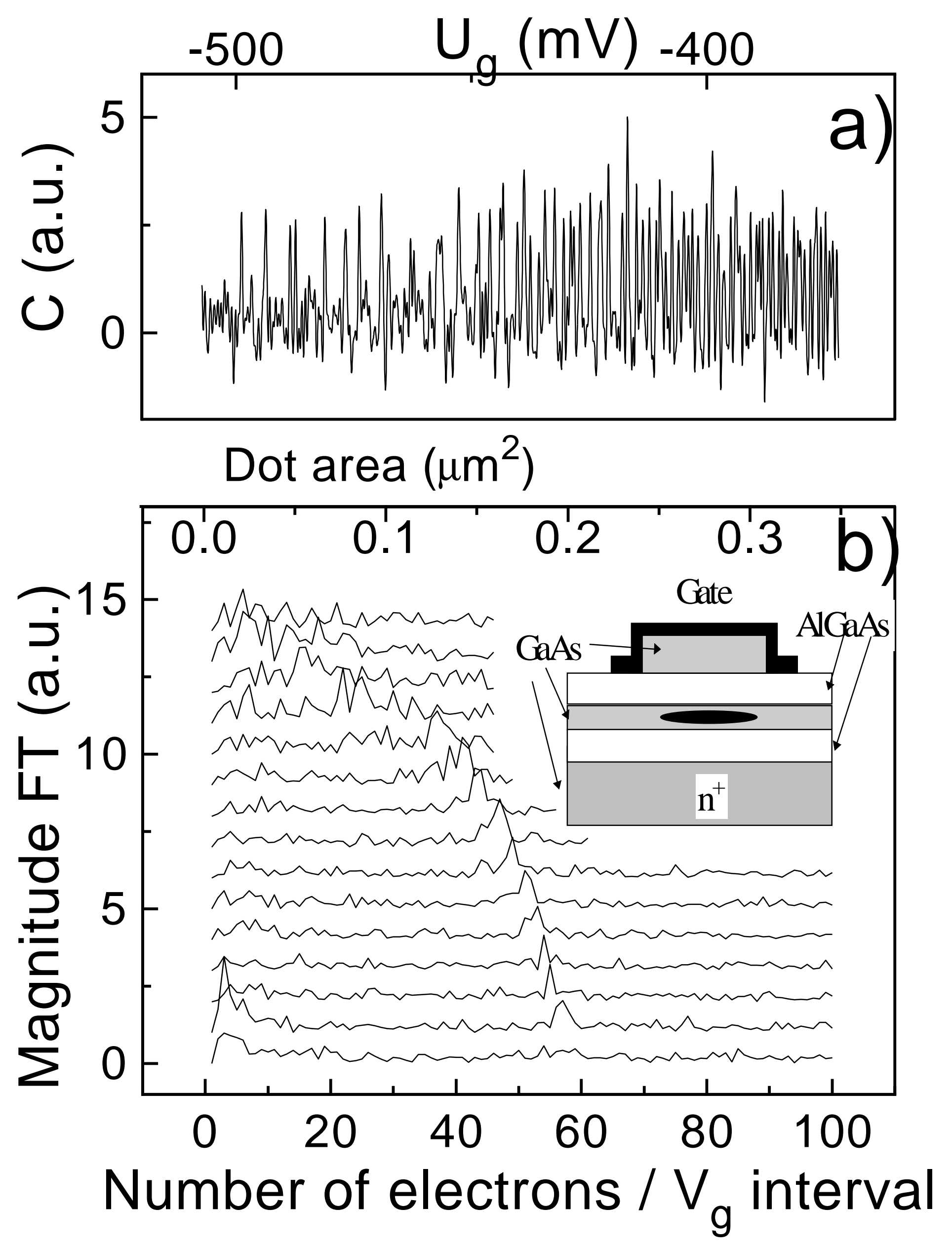


This figure "zhfig2.gif" is available in "gif" format from: http://arxiv.org/ps/cond-mat/9703241v1 